\title{
Infarto Agudo do Miocárdio: Análise do Conhecimento de Graduandos de Medicina
}

\author{
Leonardo Garcia Góes ${ }^{1}$, Marina Terumi Nakandakari ${ }^{1}$, Roberta Costa Ramos ${ }^{1}$, \\ Hélio Penna Guimarães ${ }^{2}$ \\ ${ }^{1}$ Graduandos de Medicina do Centro Universitário São Camilo \\ ${ }^{2}$ Médico especialista em Medicina de Emergência pela Associação Brasileira de Medicina de Emergência \\ (ABRAMEDE/AMB) \\ * Corresponding author. E-mail address: leonardo.goes47@gmail.com
}

\section{RESUMO}

O infarto agudo do miocárdio (IAM) é uma das principais causas de morte no Brasil sendo que a sua maioria ocorre nas primeiras 24 horas do início da doença, e quase metade delas na primeira hora. Dessa forma, a identificação precoce do quadro clínico de IAM é de suma importância para que haja um efetivo tratamento em tempo hábil. O presente estudo buscou avaliar o conhecimento desses alunos acerca do IAM, a fim de sinalizar as necessidades de preenchimento de lacunas no aprendizado médico. Trata-se de um estudo descritivo-exploratório quantitativo realizado com 260 discentes do 50 ao 120 semestre do curso de medicina de instituições brasileiras. A coleta de ocorreu por meio de aplicação de questionário com 12 questões sobre o manejo do IAM. Os dados foram analisados por meio da estatística descritiva simples. Houve prevalência do sexo feminino (66,9\%), que cursam no estado de São Paulo (86,1\%), do $50,60,70$ e 90 semestres (76,1\%), de 20 a 25 anos (83,8\%). O quinto semestre obteve média 5 , o sexto, sétimo e décimo, média 6 e o oitavo, nono, décimo primeiro e décimo segundo, média 7. As questões com mais acerto foram sobre marcadores de necrose miocárdica $(73,46 \%)$, tempo mínimo para realização do ECG (70,76\%). As com maiores taxas de erro foram sobre contraindicações de trombectomia $(13,07 \%)$ e conduta não indicada no IAM de ventrículo direito $(35,77 \%)$. Os graduandos de medicina possuem conhecimento insuficiente em relação ao manejo do infarto agudo do miocárdio.

Palavras-chave: Infarto do Miocárdio, Educação de Graduação em Medicina, Avaliação Educacional.

\section{Introdução}

As doenças cardiovasculares se tornaram a principal causa de morte em países desenvolvidos e em desenvolvimento ${ }^{1-4}$ tornando-se um problema de saúde global com proporções epidêmicas.

O infarto agudo do miocárdio (IAM) é uma das principais causas de morte no Brasil. A maioria ocorre nas primeiras 24 horas do início da doença, e quase metade delas na primeira hora ${ }^{5,6}$. Em alguns países como Franca, Portugal e Itália, a taxa de mortalidade por infarto agudo do miocárdio vem diminuindo nas últimas décadas devido a organização do atendimento, tanto pré-hospitalares quanto hospitalares, com protocolos unificados, treinamento, central de regulação e comprometimento assistencial ${ }^{5}$.

A identificação do quadro clínico de IAM por parte da população e profissionais da saúde é de suma importância para que haja um efetivo tratamento em tempo hábil. $\mathrm{O}$ atraso na procura de atendimento médico está relacionado tanto com questões relacionadas com o paciente, devido à dificuldade de reconhecimento dos sintomas e associados aos problemas na eficiência dos sistemas de saúde'1.

Oliveira e Villela $(2018)^{7}$ mostram que a importância da fase pré-hospitalar na abordagem do paciente com IAM deve-se ao fato do retardo na chegada aos serviços de saúde, em que $80 \%$ das 
vezes tem duração média superior a 2 horas, contando desde o início da apresentação dos sintomas, sendo que $50 \%$ dos óbitos por IAM foram registrados na fase pré-hospitalar.

Atualmente, há muita pouca informação na literatura sobre estudos que examinam 0 conhecimento dos alunos sobre as condições relevantes para o IAM. A literatura atual mostra que a abordagem teórica por si só não é capaz de capacitar os alunos efetivamente, contribuindo para um déficit nesse assunto emergencial8. Assim, o principal objetivo deste estudo foi avaliar o conhecimento sobre IAM em uma amostra de estudantes de medicina de diversas instituições de ensino brasileiras para identificarmos possíveis déficits no conhecimento sobre o manejo adequado do IAM.

\section{Métodos}

Trata-se de um estudo descritivo/exploratório, com abordagem quantitativa, desenvolvido com graduandos de medicina do 50 ao 120 semestre de diversas instituições de ensino superior brasileiras. Essa abrangência corresponde ao ciclo clínico onde há o primeiro contato com a cardiologia e internato onde o aluno aplica os conhecimentos adquiridos na prática. Dessa forma é possível identificar se o conhecimento acerca do manejo do IAM é consolidado desde o primeiro contato e se mantém até o final do internato.

A amostra foi definida por conveniência, conforme disponibilidade dos alunos em participar da pesquisa, obedecendo aos seguintes critérios de inclusão: estar devidamente matriculado no referido curso no período da coleta de dados e aceitar participar voluntariamente do estudo.

Os alunos foram convidados por meio de comunicação via presencial e redes sociais ficando facultada a decisão espontânea e individual de participação no estudo, sendo a amostra composta pelos que consentirem em participar do projeto, sem trazer nenhum prejuízo acadêmico ou social ao indivíduo que optar por não participar da pesquisa.

A coleta de dados ocorreu no período de fevereiro a março de 2020, após a aprovação do Comitê de Ética em Pesquisa CAAE: 23037019.1.0000.0062, Número do Parecer: 3.854.745.

O instrumento de coleta de dados foi um questionário elaborado pelos próprios pesquisadores, que foi dividido em duas partes. A primeira continha perguntas referentes ao perfil sociodemográfico da amostra, tais como idade, sexo, estado onde estuda e semestre que está cursando. A segunda parte possui 12 questões com quatro alternativas cada sobre 0 conhecimento teórico dos graduandos acerca da identificação e manejo do infarto agudo do miocárdio, construídas à luz da $\mathrm{V}$ Diretriz da Sociedade Brasileira de Cardiologia sobre Tratamento do Infarto Agudo do Miocárdio com Supradesnível do Segmento ST9.

As questões abordavam temas como o tempo para realização do eletrocardiograma na chegada do paciente ao serviço de emergência, o tempo portabalão, tempo porta-agulha, tempo de transferência para serviço com intervenção coronária percutânea primária, principal arritmia associada e seu tratamento, drogas associadas ao tratamento e redução de mortalidade, indicações para a terapia fibrinolítica, intervenção coronária percutânea primária e marcadores bioquímicos para auxílio do diagnóstico.

Os questionários foram respondidos através de formulário online, disponibilizado para os graduandos através de computadores, tablets e smartphones. Os discentes foram informados sobre os objetivos do estudo e convidados a participarem voluntariamente da pesquisa.

Por se tratar de TCLE em página WEB, e sem a possibilidade de assinatura física, após o consentimento 0 participante teve que clicar no seguinte botão para prosseguir a pesquisa: "Ao clicar no botão abaixo, o(a) Senhor(a) concorda em participar da pesquisa nos termos deste TCLE. Caso não concorde em participar, apenas feche essa página no seu navegador". Após a concordância do aluno no Termo de Consentimento Livre e Esclarecido (TCLE), em conformidade com a resolução 466/2012, procedeu-se a coleta dos dados.

As informações obtidas foram armazenadas no software aplicativo Microsoft Excel $2013 \AA$ e, posteriormente, foram analisadas de maneira descritiva, calculando-se as frequências absolutas e relativas para todas as variáveis estudadas.

Os alunos participantes puderam testar seus conhecimentos acerca do IAM desde o momento que têm contato com a cardiologia até sua formação acadêmica e identificar se estão treinados a contento neste assunto ou não. Um feedback com as respostas comentadas das questões respondidas foi fornecido ao final do questionário para que os alunos aprendam as questões que erraram. 


\section{Resultados}

A amostra foi composta por 260 graduandos, dos quais houve prevalência do sexo feminino $(66,9 \%)$, que cursam no estado de São Paulo $(86,1 \%)$, do $50,60,70$ e 90 semestres $(76,1 \%)$, de 20 a 25 anos (83,8\%). Os dados demográficos estão apresentados na Tabela 1.

Tabela 1. Dados demográficos.

\begin{tabular}{llr}
\hline \hline Variável & No & $\%$ \\
\hline \hline Faixa Etária (anos) & & \\
\hline 18 a 23 & 173 & 66,54 \\
24 a 29 & 70 & 26,93 \\
30 a 37 & 17 & 6,53 \\
\hline Sexo & & \\
\hline Masculino & 86 & 33,07 \\
Feminino & 174 & 66,93 \\
\hline Estado & & \\
\hline São Paulo & 224 & 86,15 \\
Paraná & 16 & 6,16 \\
Rio de Janeiro & 10 & 3,85 \\
Minas Gerais & 8 & 3,08 \\
Pernambuco & 1 & 0,38 \\
Mato Grosso & 1 & 0,38 \\
\hline Semestre & & \\
\hline 50 & 50 & 19,23 \\
6o & 48 & 18,46 \\
70 & 51 & 19,61 \\
80 & 17 & 6,53 \\
9o & 49 & 18,85 \\
10o & 18 & 6,93 \\
110 & 17 & 6,54 \\
120 & 10 & 3,85 \\
\hline \hline
\end{tabular}

No que concerne à avaliação geral dos graduandos acerca do conhecimento sobre IAM, a média obtida foi de 6 questões. $O$ quinto semestre obteve média 5 , o sexto, sétimo e décimo obtiveram média 6 e o oitavo, nono, décimo primeiro e décimo segundo média 7 .

As questões com maiores taxas de acerto foram em relação aos marcadores de necrose miocárdica $(73,46 \%)$ e tempo mínimo para realização do ECG $(70,76 \%)$. As que tiveram menores taxas de acerto foram com relação às contraindicações de ICP $(13,07 \%)$ e conduta não indicada em IAM de ventrículo direito (35,77\%).

Com relação ao conhecimento teórico dos discentes acerca do IAM, constatou-se que 70,76\% dos alunos afirmaram que o eletrocardiograma (ECG) deve ser realizado em até 10 minutos da chegada do paciente com suspeita de IAM. Em relação ao melhor marcador bioquímico de escolha a ser dosado na suspeita de IAM, 73,46\% responderam Troponina.

Ao serem questionados sobre a principal arritmia decorrente do IAM na fase pré-hospitalar associada a frequente causa de morte, $56,54 \%$ dos graduandos assinalaram a fibrilação ventricular e, em relação ao seu tratamento, $54,23 \%$ marcaram a desfibrilação e $32,69 \%$ o uso de Amiodarona. As respostas estão sintetizadas na Tabela 2 .

Tabela 2. Resposta referentes ao tempo para ECG, melhor marcador de necrose miocárdica, arritmia associada ao IAM e tratamento para a arritmia.

\begin{tabular}{llr}
\hline \hline Variável & No & $\%$ \\
\hline \hline Tempo para ECG (min) & & \\
\hline 5 & 36 & 13,80 \\
10 & 184 & 70,76 \\
15 & 31 & 11,92 \\
20 & 9 & 3,46 \\
\hline Melhor marcador & & \\
\hline LDH & 1 & 0,38 \\
CK & 9 & 3,46 \\
CK-MB & 59 & 22,69 \\
Troponina & 191 & 73,46 \\
\hline Arritmia do IAM & & \\
\hline Taquicardia Supraventricular & 34 & 13,08 \\
Fibrilação Atrial & 73 & 28,08 \\
Fibrilação Ventricular & 147 & 56,54 \\
Bradicardia Sinusal & 6 & 2,30 \\
\hline Tratamento da Arritmia & \multicolumn{2}{l}{} \\
\hline Amiodarona & 85 & 32,69 \\
Desfibrilação & 141 & 54,23 \\
Lidocaína & 10 & 3,85 \\
Epinefrina & 24 & 9,23 \\
\hline \hline
\end{tabular}

Quando questionados sobre o tempo para realização da intervenção coronariana primária percutânea (ICP) (tempo porta-balão), 44,23\% responderam que deve ser realizado em até 90 minutos e $27,69 \%$ e $24,61 \%$ em até 30 e 60 minutos, respectivamente. Em relação ao tempo para realização da terapia fibrinolítica (tempo portaagulha), 48,07\% responderam 30 minutos e $32,30 \%$, 60 minutos. $\mathrm{Na}$ indisponibilidade da ICP, 59,23\% responderam que 0 paciente deve ser transferido para uma instituição com sua disponibilidade em até 120 minutos e, $28,84 \%$ em até 60 minutos. As respostas estão sintetizadas na Tabela 3. 
Tabela 3. Resposta referentes aos tempos portabalão, porta-agulha e de transferência.

\begin{tabular}{lrr}
\hline \hline Variável & No & $\%$ \\
\hline \hline Tempo porta-balão (min) & & \\
\hline 10 & 9 & 3,46 \\
30 & 72 & 27,69 \\
60 & 64 & 24,61 \\
90 & 115 & 44,23 \\
\hline Tempo porta-agulha (min) & & \\
\hline 10 & 37 & 14,23 \\
30 & 125 & 48,07 \\
40 & 14 & 5,38 \\
60 & 84 & 32,30 \\
\hline Tempo de transferência (min) & & \\
\hline 120 & 154 & 59,23 \\
60 & 75 & 28,84 \\
150 & 6 & 2,30 \\
45 & 25 & 9,61 \\
\hline \hline
\end{tabular}

Em relação às terapias que reduzem mortalidade, $46,15 \%$ responderam se tratar de AAS, Clopidogrel, inibidor da enzima de conversão de angiotensina (IECA), Betabloqueador, Estatinas, enquanto $32,69 \%$ responderam Heparina, Oxigenoterapia, AAS, Clopidogrel, Estatinas, Morfina, Nitrato, Betabloqueador. Ao serem questionados sobre a conduta a ser evitada em um paciente com quadro clínico compatível com infarto agudo de ventrículo direito (IAM de VD), 35,77\% responderam expansão volêmica e $35,77 \%$ administração de Nitratos. As respostas estão sintetizadas na Tabela 4.

Tabela 4. Resposta referentes às condutas que reduzem mortalidade no IAM e contraindicações no IAM de VD.

\begin{tabular}{lrr}
\hline \hline Variável & No & $\%$ \\
\hline \hline Reduzem Mortalidade & & \\
\hline $\begin{array}{l}\mathrm{O}_{2}, \text { Clopidogrel, Morfina, Nitrato, } \\
\text { Estatinas, } \beta \text {-bloqueador, IECA }\end{array}$ & 19 & 7,31 \\
$\begin{array}{l}\text { AAS, Clopidogrel, inibidor da } \\
\text { enzima de conversão de } \\
\text { angiotensina, } \beta \text {-bloqueador, }\end{array}$ & 120 & 46,15 \\
$\begin{array}{l}\text { Estatinas } \\
\mathrm{O}_{2}, \text { AAS, Morfina, heparina, } \beta-\end{array}$ & 36 & 13,85 \\
bloqueador, IECA \\
$\begin{array}{l}\text { Heparina, O2, AAS, Clopidogrel, } \\
\text { Estatinas, Morfina, Nitrato, } \beta-\end{array}$ \\
\begin{tabular}{l} 
bloqueador \\
\hline Contraindicado no IAM de VD
\end{tabular} & 85 & 32,69 \\
\hline Nitratos & & \\
\hline
\end{tabular}

Trombolíticos

46

Expansão Volêmica

Dobutamina

93

Sobre as condutas terapêuticas, $42,30 \%$ afirmaram que pacientes com história de acidente vascular cerebral isquêmico nos últimos 3 meses têm contraindicação absoluta do uso de fibrinolíticos, $36,15 \%$ afirmou ser a presença de úlcera péptica ativa que contraindica absolutamente o procedimento, e $69,23 \%$ indicaram não ser recomendada essa conduta em IAM com duração maior que 12 horas de duração ou presença de choque cardiogênico. Em relação à não realização da ICP, 42,30\% responderam ser em caso de vaso coronário ocluído com retardo maior que 24 horas após a ocorrência do IAM, em paciente assintomático, com doença coronária de um ou dois vasos, hemodinamicamente estável e sem evidência de isquemia miocárdica a terapia. Outras respostas foram em pacientes com diagnóstico de IAM com sintomas iniciados a mais de 12 a 24 horas e evidência de arritmias ventriculares graves $(25,00 \%)$, e em pacientes com choque cardiogênico relacionado a IAM independente do retardo do início dos sintomas (19,61\%). As respostas estão sintetizadas na Tabela 5 .

Tabela 5. Resposta referentes às contraindicações de fibrinólise e de ICP.

\begin{tabular}{lrr}
\hline \hline Variável & No & \multicolumn{2}{c}{$\%$} \\
\hline \hline Contraindicação Absoluta Fibrinólise & & \\
\hline Gravidez & 45 & 17,30 \\
AVC isquêmico há 3 meses & 110 & 42,30 \\
Úlcera péptica ativa & 94 & 36,15 \\
HAS não controlada & 11 & 4,23 \\
\hline Contraindicação Fibrinólise & & \\
\hline$>12$ horas de sintoma ou choque & 180 & 69,23 \\
cardiogênico & 22 & 8,46 \\
ECG com supra de ST & 24 & 9,23 \\
Bloqueio de Ramo Novo & 34 & 13,07 \\
Impossibilidade de ICP em tempo & \\
adequado & &
\end{tabular}

Contraindicação ICP

$<12$ horas de sintoma, sem persistência de supra de ST

$34 \quad 13,07$

$>12$ a 24 horas de sintoma e arritmias ventriculares graves

$65 \quad 25,00$

$>24$ horas de sintoma, assintomático, doença coronária de $1 / 2$ vasos, estável, sem isquemia

$110 \quad 42,30$ miocárdica

Choque cardiogênico independente do tempo de início dos sintomas

$51 \quad 19,61$ 


\section{Discussão}

Foi observado no presente estudo que o número de mulheres cursando a graduação em medicina é superior ao de homens, além de maior prevalência de estudantes com 20 a 25 anos e cursando a faculdade no estado de São Paulo. Dessa forma, nosso estudo englobou perfil semelhante ao divulgado pelo Censo da Educação Superior ${ }^{10} \mathrm{em}$ relação às mesmas variáveis.

A fase pré-hospitalar na abordagem do paciente com IAM é de extrema importância uma vez que, o retardo na chegada ao serviço de saúde, muitas vezes com tempo superior a 2 horas, está associado ao aumento de mortalidade por IAM7.

A principal arritmia decorrente do IAM na fase préhospitalar associada a frequente causa de morte é a Fibrilação Ventricular (FV), sendo que o maior risco ocorre durante as primeiras $4 \mathrm{~h}$ após o início dos sintomas ${ }^{9}$. Ao serem questionados sobre qual arritmia é a mais frequente, a maioria respondeu ser a FV.

Quando a monitorização com desfibrilador manual revela ritmo de $\mathrm{FV}$, a prioridade deve ser a desfibrilação, assim que disponível, uma vez que duração da arritmia é fator prognóstico para o sucesso da desfibrilação, sendo máximo se a desfibrilação ocorrer em até 10 a 30 segundos do início da FV?.

Sobre essa conduta inicial, cerca de $55 \%$ dos alunos responderam corretamente. É de suma importância o conhecimento sobre 0 tratamento da fase pré-hospitalar, visto que tem como principal objetivo reduzir o tempo entre o início do evento isquêmico/necrose muscular até o tratamento efetivo, restaurando a perfusão miocárdica.

Além da rápida reversão da arritmia, o uso de medicações como Ácido acetilsalićlico, Clopidogrel, inibidor da enzima de conversão de angiotensina, Betabloqueador e Estatinas também está associada com redução de mortalidade ${ }^{9}$, o que foi corretamente indicado por cerca de $46 \%$ dos estudantes.

O Ácido acetilsalicílico é o antiplaquetário de eleição a ser utilizado e deve ser combinado ao Clopidogrel em pacientes que receberam terapia trombolítica inicial, demonstrando seu benefício em reduzir eventos cardiovasculares maiores. Os IECA são úteis após IAM com disfunção ventricular esquerda, com ou sem sintomas, sendo mais efetivos nos subgrupos de maior risco, como disfunção ventricular sintomática, infarto de parede anterior, taquicardia e antecedente de infarto prévio9 ${ }^{9}$
$O$ uso de betabloqueadores reduz a frequência cardíaca, a pressão arterial e o inotropismo, reduzindo o consumo de oxigênio pelo miocárdio, melhorando sua perfusão; tais ações são responsáveis por reduzir as taxas de ruptura miocárdica, limitar o tamanho do infarto e melhorar a função cardíaca. A administração de estatinas, principalmente com relação à precocidade (primeiras 24 horas) da introdução, demostrou redução de 25\% na mortalidade em 1 ano após IAM9.

O objetivo da avaliação inicial da vítima de IAM é a detecção imediata dos sinais clínicos sugestivos desse quadro. Nesse sentido, a primeira conduta a ser tomada na suspeita de IAM é a realização de Eletrocardiograma (ECG). O ECG de 12 derivações deve ser realizado pela equipe médica em até 10 minutos da chegada do paciente ao serviço9. A maioria dos alunos responderam corretamente a essa questão.

Além da realização de ECG, outro exame utilizado para auxílio diagnóstico e prognóstico é a mensuração dos marcadores bioquímicos de lesão miocárdica que devem ser realizados em todo paciente com suspeita de IAM. Esses marcadores são liberados na corrente sanguínea quando as células miocárdicas são irreversivelmente danificadas ${ }^{11}$ e são úteis para confirmar o diagnóstico de IAM em pacientes com quadro sugestivo de síndrome coronariana aguda, onde o diagnóstico de IAM não está estabelecido. Além do diagnóstico, os marcadores fornecem importantes informações prognósticas, por existir uma associação direta entre a elevação dos mesmos e o risco de eventos cardíacos em curto e médio prazos ${ }^{12,13}$.

Dentre os marcadores estão as troponinas, Isoenzima $\mathrm{MB}$ da creatinoquinase (CK-MB) e a mioglobina. Em comparação, a troponina possui maior especificidade para lesão miocárdica, enquanto a CK-MB é encontrada em tecidos não cardíacos; Além disso, a troponina é capaz de detectar lesão miocárdica de pequena extensão, o que não ocorre com a CK-MB14.

A CK MB e a mioglobina são marcadores precoces de lesão miocárdica. No entanto, por não ser um marcador cardioespecífico, a principal vantagem das suas mensurações parece ser na detecção de IAM nas primeiras horas de evolução?

Dessa forma, as troponinas são os marcadores bioquímicos de escolha detecção de necrose miocárdica, sendo que a CK-MB pode ser utilizada se troponinas não estiverem disponíveis9. $\mathrm{Na}$ questão que abordava qual o melhor marcador bioquímico de escolha a ser dosado na suspeita de 
infarto agudo do miocárdio, a maioria respondeu corretamente ser a troponina.

De acordo com a V Diretriz da Sociedade Brasileira de Cardiologia sobre tratamento do infarto agudo do miocárdio com supradesnível do segmento ST, a primeira conduta a ser realizada após o diagnóstico de IAM é a recanalização coronariana através da fibrinólise em no máximo 30 minutos ou intervenção coronária percutânea primária (ICP) em até 90 minutos do diagnóstico. $\mathrm{Na}$ indisponibilidade da ICP pode-se transferir o paciente para uma instituição com sua disponibilidade se o tempo de transferência previsto não exceder 120 minutos $^{9}$. Ao serem questionados sobre esses tempos para intervenção, a maioria respondeu corretamente.

Sobre essas terapias, a ICP está indicada em pacientes com IAM com sintomas iniciados < 12 horas, com persistência de elevação do segmento ST ou evidência de bloqueio de ramo recente, realizada até 90 minutos após o diagnóstico, com sintomas iniciados > 12 a 24 horas e/ou evidência de isquemia miocárdica persistente ou instabilidade hemodinâmica/arritmias ventriculares graves, recanalização de vaso coronário ocluído com retardo > 24 horas após a ocorrência do IAM, em paciente assintomático, com doença coronária de um ou dois vasos, hemodinamicamente estável e sem evidência de isquemia miocárdica ${ }^{15,16 .}$

Ao serem questionados sobre essas situações, a maioria dos estudantes respondeu uma das três alternativas que apresentavam situações em que a terapia era contraindicada.

Uma alternativa terapêutica para reperfusão coronariana é o uso de fibrinolíticos que são indicados nos pacientes com sintomas sugestivos de IAM com duração > 20 minutos e $<12$ horas não responsiva a nitrato sublingual, supradesnivelamento do $\mathrm{ST}>1 \mathrm{~mm}$ em pelo menos duas derivações precordiais contíguas ou duas periféricas adjacentes, bloqueio de ramo novo, impossibilidade de realizar ICP em tempo adequado ${ }^{17}$. Ao serem questionados sobre essas indicações, a maioria dos estudantes responderam corretamente.

Questionou-se também quanto as ocasiões em que essa terapia é contraindicada. Constituem-se contraindicações absolutas: sangramento intracraniano prévio, acidente vascular cerebral isquêmico nos últimos 3 meses, dano ou neoplasia no sistema nervoso central, trauma significante na cabeça ou rosto nos últimos 3 meses, sangramento ativo ou diátese hemorrágica, qualquer lesão vascular cerebral conhecida, dissecção aguda de aorta, discrasia sanguínea ${ }^{9}$.
A questão apresentava como resposta correta a presença de acidente vascular cerebral isquêmico nos últimos 3 meses como contraindicação absoluta. As outras condições contraindicavam relativamente a terapia. Menos da metade dos alunos responderam corretamente.

Outra questão abordada foi o manejo do IAM de ventrículo direito (VD). Seu tratamento inclui manutenção precoce da pré-carga, redução da póscarga do VD, suporte inotrópico para o VD e reperfusão precoce. Por sua influência na pré-carga, os nitratos e diuréticos podem reduzir o débito cardíaco e provocar hipotensão grave, se o VD estiver isquêmico. Nessas situações, geralmente uma expansão volêmica, com solução salina fisiológica, normaliza a hipotensão e melhora o débito cardíaco. Em outros casos, porém, a sobrecarga de volume pode ocasionar elevação acentuada da pressão de enchimento do VD e o consequente agravamento da dilatação ventricular, com redução do débito cardíaco. Nesses casos, o suporte inotrópico com dobutamina deve ser iniciado imediatamente. A terapia fibrinolítica e a ICP primária com reperfusão subsequente têm demonstrado aumentar a fração de ejeção do VD e reduzir a incidência de bloqueio átrio-ventricular completo9. Apenas 35\% dos alunos demostraram conhecimento sobre o tratamento preconizado para o infarto de VD.

Espera-se que os estudantes de semestres mais avançados tenham uma maior chance de obter um desempenho melhor em relação aos alunos em semestres menores, devido ao conhecimento que deve ser cumulativo ao longo dos semestres, porém após as análises dos dados, percebe-se que os graduandos, dos semestres mais adiantados obtiveram resultado semelhante aos dos semestres anteriores.

Esses dados apontam para uma lacuna em alguns quesitos na formação do médico e evidenciam a importância da discussão teórica sobre IAM durante o curso de graduação em medicina, e necessita de complementação com cursos de formação, capacitação e educação permanente.

Nessa perspectiva, sugere-se que as instituições de ensino, junto com a equipe de docentes reavalie a grade curricular do curso, acrescentando disciplinas obrigatórias ou optativas que trate de temas relacionados ao IAM, as quais devem apresentar informações sobre a doença, o seu manejo clínico, as manifestações clínicas, medidas terapêuticas, entre outras questões. 


\section{Conclusão}

Este estudo permitiu concluir que os graduandos possuem déficit de conhecimento principalmente no que tange ao tempo porta-balão, porta-agulha, terapias que reduzem mortalidade, contraindicação à fibrinólise, contraindicações de ICP e conduta não indicada em IAM de ventrículo direito. Além disso percebeu-se que os graduandos dos semestres mais adiantados obtiveram resultado semelhante aos dos semestres anteriores.

Os dados apontam para a importância da discussão teórica sobre IAM durante o curso de graduação em medicina e necessidade de complementação com cursos de formação, capacitação e educação permanente. Dessa forma, sugere-se uma revisão da grade curricular a fim de incluir estratégias para a aquisição de um conhecimento mais duradouro a cerca de um tema tão importante no cotidiano da emergência.

\section{Referências}

1. Passinho RS, Sipolatti WGR, Fioresi M, Primo CC. Sinais, sintomas e complicações do infarto agudo do miocárdio. Rev. enferm. UFPE on-line, 2018;

247-264.

https://pesquisa.bvsalud.org/portal/resource/pt/bibli o-947058

2. Santos JD, Meira KC, Camacho AR, Salvador PTCDO, Guimarães RM, Pierin ÂMG, Freire FHMDA. Mortalidade por infarto agudo do miocárdio no Brasil e suas regiões geográficas: análise do efeito da idade-período-coorte. Ciência \& Saúde Coletiva, 23. 2018; 1621-1634. https://www.scielosp.org/article/csc/2018.v23n5/16 $21-1634 / \mathrm{pt} /$

3. Bin LL. Risk Factor Differences in Acute Myocardial Infarction between young and older people: A systematic review and meta-analysis. International Journal of Cardiovascular Sciences, (AHEAD). 2019.

4. Milano SS, Moura Júnior OVD, Bordin AAS, Marques GL. C-reactive protein is a predictor of mortality in ST-segment elevation acute myocardial infarction. International Journal of Cardiovascular Sciences, 32(2). 2019; 118-124. https://www.scielo.br/j/ijcs/a/wNpWQZyK3jskCrmd h8x7t3p/?lang=en

5. Baruzzi ACDA, Stefanini E, Pispico A. Infarto agudo do miocárdio com supra de ST: trombólise em qualquer local que a medicação esteja disponível. Rev. Soc. Cardiol. Estado de Säo Paulo. 2018; 409-420. https://pesquisa.bvsalud.org/portal/resource/pt/bi blio-970540

6. Hoepfner C, Roma E, Lana JV, Santin AL, Borga AL, Yamamoto AC, Techentin JV. Knock on the right door. How we are treating the patient with acute myocardial infarction. International Journal of Cardiovascular Sciences, 31(5). 2018; 520526. https://www.scielo.br/j/ijcs/a/rGW5PY9kNfT x3srdRMWRnbr/?lang=en

7. Oliveira GMMD, Villela PB. The Importance of the Prehospital Phase in ST Elevation Myocardial Infarction. Arquivos brasileiros de cardiologia, 111(4). 2018; 594-595. https://www.scielo.br/j/abc/a/sdvK9sZss3V7pgSc cMpgmLx/?lang=en

8. Miranda GNB, Ribeiro LLPA, Ibiapaba MCC, Rodrigues IC, Lourenço RP, Messias KLM. Análise do Conhecimento e Preparação de Estudantes de Medicina Acerca do Infarto Agudo do Miocárdio (IAM) e sua Abordagem Emergencial. In: VI congresso brasileiro de medicina de emergência adulto e pediátrico, 6 ., Ceará. Anais [...] . Fortaleza: Abramede, 2018. v. 1. http://abramede2018.web15f60.uni5.net/anais/ edicao-atual

9. Piegas LS, Timerman A, Feitosa GS, Nicolau JC, Mattos LAP, Andrade MD, Mathias Junior W. V Diretriz da Sociedade Brasileira de Cardiologia sobre tratamento do infarto agudo do miocárdio com supradesnível do segmento ST. Arquivos brasileiros de cardiologia, 105(2). 2015; 1-121. https://www.scielo.br/j/abc/a/VPF5J5cmYSyFFfM $8 \mathrm{Xfd} 7 \mathrm{dkf} /$ ?lang=pt

10.da Educação Superior C. Ministério da Educação. Brasília: Instituto Nacional de Estudos e Pesquisas Educacionais Anísio Teixeira. 2015. http://www.schwartzman.org.br/simon/pdf/geres.p df

11. Apple FS. Tissue specificity of cardiac troponin I, cardiac troponin $\mathrm{T}$ and creatine kinase-MB. Clinica chimica acta, 284(2). 1999; 151-159. https://www.sciencedirect.com/science/article/abs /pii/S0009898199000777

12. Ferguson JL, Beckett GJ, Stoddart M, Walker SW, Fox KAA. Myocardial infarction redefined: the new ACC/ESC definition, based on cardiac troponin, increases the apparent incidence of infarction. Heart, 88(4). 2002; 343-347. https://heart.bmj.com/content/88/4/343.short

13. Koukkunen $\mathrm{H}$, Penttilä K, Kemppainen A, Penttilä I, Halinen MO, Rantanen T, Pyörälä K. 
Differences in the diagnosis of myocardial infarction by troponin $\mathrm{T}$ compared with clinical and epidemiologic criteria. The American journal of cardiology, 88(7). 2001; 727-731. https://www.sciencedirect.com/science/article/abs /pii/S0002914901018410

14. Apple FS, Wu AH, Jaffe AS, Panteghini $M$, Christenson RH, NACB COMMITTEE MEMBERS, Mair J. National Academy of Clinical Biochemistry and IFCC Committee for Standardization of Markers of Cardiac Damage Laboratory Medicine practice guidelines: analytical issues for biomarkers of heart failure. Circulation, 116(5). 2007; e95-e98. https://www.ahajournals.org/doi/full/10.1161/CIR CULATIONAHA.107.185266

15. Andersen HR, Nielsen TT, Vesterlund T, Grande $\mathrm{P}$, Abildgaard $\mathrm{U}$, Thayssen $\mathrm{P}$, DANAMI-2 Investigators. Danish multicenter randomized study on fibrinolytic therapy versus acute coronary angioplasty in acute myocardial infarction: rationale and design of the DANish trial in Acute Myocardial Infarction-2 (DANAMI-2). American heart journal, 146(2). 2003; 234-241. https://www.sciencedirect.com/science/article/abs /pii/S0002870303003168

16. Jolly S, Newton G, Horlick E, Seidelin PH, Ross HJ, Husain M, Dzavik V. Effect of vasopressin on hemodynamics in patients with refractory cardiogenic shock complicating acute myocardial infarction. The American journal of cardiology, 96(12). 2005; 1617-1620. https://www.sciencedirect.com/science/article/abs /pii/S0002914905015833

17. Feitosa GC, Albuquerque D, Nicolau JC. II Diretriz da Sociedade Brasileira de Cardiologia para o tratamento do infarto agudo do miocárdio. Arq Bras Cardiol, 74(Supl II). 2000; 1-46.

\section{Informações sobre o Artigo}

Recebido: 02/06/2021

Aceito: 26/07/2021

Conflitos de interesse: nenhum.

Agências financiadoras: nenhuma. 